1 Hacettepe Journal of Mathematics and Statistics

$\bigcap$ Volume 45 (2) (2016), 337-342

\title{
On conditions for univalence of some integral operators
}

\author{
Daniel Breaz* and Virgil Pescar ${ }^{\dagger}$
}

\begin{abstract}
In this paper, we obtain new univalence conditions for the integral operators $F_{[|\delta|]}(z)$ and $G_{[|\delta|]}(z)$ of analytic functions defined in the open unit disk.
\end{abstract}

Keywords: Integral operator, univalence, integer part, modulus.

2000 AMS Classification: 30C45.

Received: 07.02.2013 Accepted: 02.03.2015 Doi : 10.15672/HJMS.20164512496

\section{Introduction}

Let $\mathcal{A}$ denote the class of functions of the form $f(z)=z+a_{2} z^{2}+\ldots$ which are analytic in the open unit disc $\mathcal{U}=\{z:|z|<1\}$. Further, by $\mathcal{S}$ we shall denote the class of all functions in $\mathcal{A}$ which are univalent in $\mathcal{U}$.

Pescar [7], has obtained the following univalence criteria

1.1 Theorem. [7] Let $\gamma \in \mathbb{C}, f \in \mathcal{S}, f(z)=z+a_{2} z^{2}+\ldots$

If

$$
\left|\frac{z f^{\prime}(z)-f(z)}{z f(z)}\right| \leq 1, \forall z \in \mathcal{U}
$$

and

$$
|\gamma| \leq \frac{1}{\max _{|z| \leq 1}\left[\left(1-|z|^{2}\right) \cdot|z| \cdot \frac{|z|+\left|a_{2}\right|}{1+\left|a_{2}\right||z|}\right]}
$$

then

$$
F_{\gamma}(z)=\int_{0}^{z}\left(\frac{f(t)}{t}\right)^{\gamma} d t
$$

*Department of Mathematics, "1 Decembrie 1918" University of Alba Iulia, Faculty of Science, 510009 Alba Iulia, Romania.

Email : dbreaz@uab.ro

†Department of Mathematics, "Transilvania" University of Braşov, Faculty of Mathematics and Computer Science, 500091 Braşov, Romania.

Email : virgilpescar@unitbv.ro 
is in the class $\mathcal{S}$.

1.2 Theorem. [7] Let $\alpha, \beta, \gamma \in \mathbb{C}, f \in \mathcal{S}, f(z)=z+a_{2} z^{2}+\ldots$

If

$$
\begin{aligned}
& \left|\frac{z f^{\prime}(z)-f(z)}{z f(z)}\right| \leq 1, \forall z \in \mathcal{U}, \\
& \operatorname{Re} \beta \geq \operatorname{Re} \alpha>0
\end{aligned}
$$

and

$$
|\gamma| \leq \frac{1}{\max _{|z| \leq 1}\left[\frac{1-|z|^{2 \operatorname{Re} \alpha}}{\operatorname{Re} \alpha} \cdot|z| \cdot \frac{|z|+\left|a_{2}\right|}{1+\left|a_{2}\right||z|}\right]}
$$

then

$$
G_{\beta, \gamma}(z)=\left[\beta \int_{0}^{z} t^{\beta-1}\left(\frac{f(t)}{t}\right)^{\gamma} d t\right]^{\frac{1}{\beta}}
$$

is in the class $\mathcal{S}$.

We define the next two integral operators

$$
F_{[|\delta|]}(z)=\int_{0}^{z}\left(\frac{f_{1}(t)}{t}\right)^{\alpha_{1}} \cdot \ldots \cdot\left(\frac{f_{[|\delta|]}(t)}{t}\right)^{\alpha_{[|\delta|]}} d t,
$$

where $\delta \in \mathbb{C},|\delta| \notin[0,1), \alpha_{i} \in \mathbb{C}, f_{i} \in \mathcal{A}, i=\overline{1,[|\delta|]}, \alpha_{1} \cdot \ldots \cdot \alpha_{[|\delta|]}=\delta$ and

$$
G_{[|\gamma|]}(z)=\left[\gamma \int_{0}^{z} t^{\gamma-1}\left(\frac{f_{1}(t)}{t}\right)^{\alpha_{1}} \cdot \ldots \cdot\left(\frac{f_{[|\gamma|]}(t)}{t}\right)^{\alpha_{[|\gamma|]}} d t\right]^{\frac{1}{\gamma}}
$$

$\gamma \in \mathbb{C},|\gamma| \notin[0,1), \alpha_{i} \in \mathbb{C}, f_{i} \in \mathcal{A}, i=\overline{1,[|\gamma|]}, \alpha_{1} \cdot \ldots \cdot \alpha_{[|\gamma|]}=\gamma$.

In this paper, we obtain new univalence conditions for the integral operators $F_{[|\delta|]}(z)$ and $G_{[|\delta|]}(z)$.

\section{Preliminary results}

In order to derive our main results, we have to recall here the following lemmas:

2.1 Lemma. [2] If the function $f$ is regular in unit disk $\mathcal{U}, f(z)=z+a_{2} z^{2}+\ldots$ and

$$
\left(1-|z|^{2}\right)\left|\frac{z f^{\prime \prime}(z)}{f^{\prime}(z)}\right| \leq 1,
$$

for all $z \in \mathcal{U}$, then the function $f$ is univalent in $\mathcal{U}$.

2.2 Lemma. [5] Let $\alpha$ be a complex number, $\operatorname{Re} \alpha>0$ and $f(z)=z+a_{2} z^{2}+\ldots$ be $a$ regular function in $\mathrm{U}$. If

$$
\frac{1-|z|^{2 \operatorname{Re} \alpha}}{\operatorname{Re} \alpha}\left|\frac{z f^{\prime \prime}(z)}{f^{\prime}(z)}\right| \leq 1,
$$

for all $z \in \mathcal{U}$, then for any complex number $\beta, \operatorname{Re} \beta \geq \operatorname{Re} \alpha$, the function

$$
F_{\beta}(z)=\left[\beta \int_{0}^{z} t^{\beta-1} f^{\prime}(t) d t\right]^{\frac{1}{\beta}}
$$

is in the class $\mathcal{S}$. 
2.3 Lemma. [3] If the function $g$ is regular in $\mathcal{U}$ and $|g(z)|<1$ in $\mathcal{U}$, then for all $\xi \in \mathcal{U}$, the following inequalities hold

$$
\left|\frac{g(\xi)-g(z)}{1-\overline{g(z)} g(\xi)}\right| \leq\left|\frac{\xi-z}{1-\bar{z} \xi}\right|(2.1)
$$

and

$$
\left|g^{\prime}(z)\right| \leq \frac{1-|g(z)|^{2}}{1-|z|^{2}},
$$

the equalities hold in the case $g(z)=\epsilon \frac{z+u}{1+\bar{u} z}$, where $|\epsilon|=1$ and $|u|<1$.

2.4 Remark. [3] For $z=0$, from inequality (2.1) we obtain for every $\xi \in \mathcal{U}$,

$$
\left|\frac{g(\xi)-g(0)}{1-\overline{g(0)} g(\xi)}\right| \leq|\xi|
$$

and hence,

$$
|g(\xi)| \leq \frac{|\xi|+|g(0)|}{1+\overline{g(0)} g(\xi)} .
$$

Considering $g(0)=a$ and $\xi=z$, then

$$
|g(z)| \leq \frac{|z|+|a|}{1+|a||z|},
$$

for all $z \in \mathcal{U}$.

\section{Main results}

3.1 Theorem. Let $M>1, \delta \in \mathbb{C},|\delta| \notin[0,1), \alpha_{i} \in \mathbb{C}$, for $i=\overline{1,[|\delta|]}$ and $\alpha_{1} \cdot \ldots \cdot \alpha_{[|\delta|]}=$ $\delta$. If $f_{i} \in \mathcal{A}, f_{i}(z)=z+a_{2}^{i} z^{2}+\ldots$, for $i=\overline{1,[|\delta|]}$ and

$$
\begin{aligned}
& \left|\frac{z f_{i}^{\prime}(z)-f_{i}(z)}{z f_{i}(z)}\right| \leq 1, \forall i=\overline{1,[|\delta|]}, z \in \mathcal{U},(3.1) \\
& \frac{\left|\alpha_{1}\right|+\ldots+\left|\alpha_{[|\delta|]}\right|}{\left|\alpha_{1} \cdot \ldots \cdot \alpha_{[|\delta|]}\right|} \leq M,(3.2) \\
& \left|\alpha_{1} \cdot \ldots \cdot \alpha_{[|\delta|]}\right| \leq \frac{1}{M \max _{|z| \leq 1}\left[\left(1-|z|^{2}\right) \cdot|z| \cdot \frac{|z|+|c|}{1+|c||z|}\right]},
\end{aligned}
$$

where

$$
|c|=\frac{\left|\alpha_{1} a_{2}^{1}+\ldots+\alpha_{[|\delta|]} a_{2}^{[|\delta|]}\right|}{M\left|\alpha_{1} \cdot \ldots \cdot \alpha_{[|\delta|]}\right|},
$$

then

$$
F_{[|\delta|]}(z)=\int_{0}^{z}\left(\frac{f_{1}(t)}{t}\right)^{\alpha_{1}} \cdot \ldots \cdot\left(\frac{f_{[|\delta|]}(t)}{t}\right)^{\alpha_{[|\delta|]}} d t
$$

is in the class $\mathcal{S}$.

Proof. We have $f_{i} \in \mathcal{A}$, for all $i=\overline{1,[|\delta|]}$ and $\frac{f_{i}(z)}{z} \neq 0$, for all $i=\overline{1,[|\delta|]}$.

Let $g$ be the function $g(z)=\left(\frac{f_{1}(z)}{z}\right)^{\alpha_{1}} \cdot \ldots \cdot\left(\frac{f_{[|\delta|]}(z)}{z}\right)^{\alpha_{[|\delta|]}}, z \in \mathcal{U}$. We have $g(0)=1$.

Consider the function

$$
h(z)=\frac{1}{M\left|\alpha_{1} \cdot \ldots \cdot \alpha_{[|\delta|]}\right|} \cdot \frac{F_{[|\delta|]}^{\prime \prime}(z)}{F_{[|\delta|]}^{\prime}(z)}, z \in \mathcal{U} .
$$


The function $h(z)$ has the form:

$$
h(z)=\frac{1}{M\left|\alpha_{1} \cdot \ldots \cdot \alpha_{[|\delta|]}\right|} \sum_{i=1}^{[|\delta|]} \alpha_{i} \frac{z f_{i}^{\prime}(z)-f_{i}(z)}{z f_{i}(z)} .
$$

Also,

$$
h(0)=\frac{1}{M\left|\alpha_{1} \cdot \ldots \cdot \alpha_{[|\delta|]}\right|} \sum_{i=1}^{[|\delta|]} \alpha_{i} a_{2}^{i}
$$

By using the relations (3.1) and (3.2) we obtain that $|h(z)|<1$ and

$$
|h(0)|=\frac{\left|\alpha_{1} a_{2}^{1}+\ldots+\alpha_{[|\delta|]} a_{2}^{[|\delta|]}\right|}{M\left|\alpha_{1} \cdot \ldots \cdot \alpha_{[|\delta|]}\right|}=|c| .
$$

Applying Remark 2.4 for the function $h$ we obtain

$$
\frac{1}{M\left|\alpha_{1} \cdot \ldots \cdot \alpha_{[|\delta|]}\right|} \cdot\left|\frac{F_{[|\delta|]}^{\prime \prime}(z)}{F_{[|\delta|]}^{\prime}(z)}\right| \leq \frac{|z|+|c|}{1+|c||z|}, \forall z \in \mathcal{U}
$$

and

$$
\left|\left(1-|z|^{2}\right) \cdot z \cdot \frac{F_{[|\delta|]}^{\prime \prime}(z)}{F_{[|\delta|]}^{\prime}(z)}\right| \leq M\left|\alpha_{1} \cdot \ldots \cdot \alpha_{[|\delta|]}\right|\left(1-|z|^{2}\right) \cdot|z| \cdot \frac{|z|+|c|}{1+|c||z|}, \forall z \in \mathcal{U} .(3.4)
$$

Consider the function $H:[0,1] \rightarrow \mathbb{R}$ defined by

$$
H(x)=\left(1-x^{2}\right) x \frac{x+|c|}{1+|c| x} ; x=|z| .
$$

We have

$$
H\left(\frac{1}{2}\right)=\frac{3}{8} \cdot \frac{1+2|c|}{2+|c|}>0 \Rightarrow \max _{x \in[0,1]} H(x)>0 .
$$

Using this result and from (3.4) we have:

$$
\left|\left(1-|z|^{2}\right) \cdot z \cdot \frac{F_{[|\delta|]}^{\prime \prime}(z)}{F_{[|\delta|]}^{\prime}(z)}\right| \leq M\left|\alpha_{1} \cdot \ldots \cdot \alpha_{[|\delta|]}\right| \cdot \max _{|z|<1}\left[\left(1-|z|^{2}\right) \cdot|z| \cdot \frac{|z|+|c|}{1+|c||z|}\right], \forall z \in \mathcal{U} .(3.5)
$$

Applying the condition (3.3) in the form (3.5) we obtain that

$$
\left(1-|z|^{2}\right) \cdot\left|z \cdot \frac{F_{[|\delta|]}^{\prime \prime}(z)}{F_{[|\delta|]}^{\prime}(z)}\right| \leq 1, \forall z \in \mathcal{U}
$$

and from Lemma 2.1 we obtain that $F_{[|\delta|]} \in \mathcal{S}$.

3.2 Theorem. Let $M>1, \gamma, \delta \in \mathbb{C},|\gamma| \notin[0,1), \alpha_{i} \in \mathbb{C}$, for $i=\overline{1,[|\gamma|]}, \alpha_{1} \cdot \ldots \cdot \alpha_{n}=$ $[|\gamma|]$. If $f_{i} \in \mathcal{A}, f_{i}(z)=z+a_{2}^{i} z^{2}+\ldots$, for $i=\overline{1,[|\gamma|]}$ and

$$
\left|\frac{z f_{i}^{\prime}(z)-f_{i}(z)}{z f_{i}(z)}\right| \leq 1, \forall i=\overline{1,[|\gamma|]}, z \in \mathcal{U},(3.6)
$$




$$
\begin{aligned}
& \frac{\left|\alpha_{1}\right|+\ldots+\left|\alpha_{[|\gamma|]}\right|}{\left|\alpha_{1} \cdot \ldots \cdot \alpha_{[|\gamma|]}\right|} \leq M,(3.7) \\
& \operatorname{Re} \gamma \geq \operatorname{Re} \delta>0 \\
& \left|\alpha_{1} \cdot \ldots \cdot \alpha_{[|\gamma|]}\right| \leq \frac{1}{M \max _{|z| \leq 1}\left[\left(1-|z|^{2}\right) \cdot|z| \cdot \frac{|z|+|c|}{1+|c||z|}\right]}
\end{aligned}
$$

where

$$
|c|=\frac{\left|\alpha_{1} a_{2}^{1}+\ldots+\alpha_{[|\gamma|]} a_{2}^{[|\gamma|]}\right|}{M\left|\alpha_{1} \cdot \ldots \cdot \alpha_{[|\gamma|]}\right|},
$$

then

$$
G_{[|\gamma|]}(z)=\left[\gamma \int_{0}^{z} t^{\gamma-1}\left(\frac{f_{1}(t)}{t}\right)^{\alpha_{1}} \cdot \ldots \cdot\left(\frac{f_{[|\gamma|]}(t)}{t}\right)^{\alpha_{[|\gamma|]}} d t\right]^{\frac{1}{\gamma}}
$$

is in the class $\mathcal{S}$.

Proof. We consider the function

$$
h(z)=\int_{0}^{z}\left(\frac{f_{1}(t)}{t}\right)^{\alpha_{1}} \cdot \ldots \cdot\left(\frac{f_{[|\gamma|]}(t)}{t}\right)^{\alpha_{[|\gamma|]}} d t .
$$

Define the function

$$
p(z)=\frac{1}{M\left|\alpha_{1} \cdot \ldots \cdot \alpha_{[|\gamma|]}\right|} \cdot \frac{h^{\prime \prime}(z)}{h^{\prime}(z)}, z \in \mathcal{U} .
$$

The function $p(z)$ has the form:

$$
p(z)=\frac{1}{M\left|\alpha_{1} \cdot \ldots \cdot \alpha_{[|\gamma|]}\right|} \sum_{i=1}^{[|\gamma|]} \alpha_{i} \frac{z f_{i}^{\prime}(z)-f_{i}(z)}{z f_{i}(z)} .
$$

By using the relations (3.6) and (3.7) we obtain $|p(z)|<1$ and

$$
|p(0)|=\frac{\left|\alpha_{1} a_{2}^{1}+\ldots+\alpha_{[|\gamma|]} a_{2}^{[|\gamma|]}\right|}{M\left|\alpha_{1} \cdot \ldots \cdot \alpha_{[|\gamma|]}\right|}=|c| .
$$

Applying Remark 2.4 for the function $h$ we obtain

$$
\frac{1}{M\left|\alpha_{1} \cdot \ldots \cdot \alpha_{[|\gamma|]}\right|} \cdot\left|\frac{h^{\prime \prime}(z)}{h^{\prime}(z)}\right| \leq \frac{|z|+|c|}{1+|c||z|}, \forall z \in \mathcal{U}
$$

and

$$
\left|\frac{1-|z|^{2 \operatorname{Re} \delta}}{\operatorname{Re} \delta} \cdot z \cdot \frac{h^{\prime \prime}(z)}{h^{\prime}(z)}\right| \leq M\left|\alpha_{1} \cdot \ldots \cdot \alpha_{[|\gamma|]}\right| \frac{1-|z|^{2 \operatorname{Re} \delta}}{\operatorname{Re} \delta} \cdot|z| \cdot \frac{|z|+|c|}{1+|c||z|}, \forall z \in \mathcal{U} .(3.9)
$$

Consider the function $Q:[0,1] \rightarrow \mathbb{R}$ defined by

$$
Q(x)=\frac{1-x^{2 \operatorname{Re} \delta}}{\operatorname{Re} \delta} \cdot x \cdot \frac{x+|c|}{1+|c| x} ; x=|z| .
$$

We have $Q\left(\frac{1}{2}\right)>0 \Rightarrow \max _{x \in[0,1]} Q(x)>0$. 
Using this result in (3.9), we have:

$$
\frac{1-|z|^{2 \operatorname{Re} \delta}}{\operatorname{Re} \delta}\left|\frac{z h^{\prime \prime}(z)}{h^{\prime}(z)}\right| \leq M\left|\alpha_{1} \cdot \ldots \cdot \alpha_{[|\gamma|]}\right| \cdot \max _{|z|<1}\left[\frac{1-|z|^{2 \operatorname{Re} \delta}}{\operatorname{Re} \delta} \cdot|z| \cdot \frac{|z|+|c|}{1+|c||z|}\right], \forall z \in \mathcal{U} .
$$

Applying the condition (3.8) in the relation (3.10), we obtain that

$$
\frac{1-|z|^{2 \operatorname{Re} \delta}}{\operatorname{Re} \delta}\left|\frac{z h^{\prime \prime}(z)}{h^{\prime}(z)}\right| \leq 1, \forall z \in \mathcal{U}
$$

and from Lemma 2.2, we obtain that $G_{[|\gamma|]} \in \mathcal{S}$.

\section{References}

[1] D. Breaz, N. Breaz, Two integral operators, Studia Universitatis "Babeş-Bolyai", Mathematica, Cluj-Napoca, No.3, 2002, 13-21.

[2] J. Becker, Lownersche Differntialgleichung und quasikonform fortsetzbare schlichte Functionen, J. Reine Angew. Math. 255(1972), 23-43.

[3] G. M. Goluzin, Geometriceskaia teoria funktii kompleksnogo peremenogo, Moscova, 1966.

[4] Y.J. Kim, E.P. Merkes, On an integral of powers of a spirallike function, Kyungpook Math., J., 12 (1972), 2, 249-253.

[5] N.N. Pascu, An improvement of Becker's Univalence Criterion, Proceedings of the Commemorative Session Simion Stoilow, University of Braşov, 1987, 43-48.

[6] N.N. Pascu, V.Pescar, On the integral operators of Kim-Merkes and Pfaltzgraff, Mathematica, Univ. "Babeş-Bolyai", Cluj-Napoca, 32 (55), 2(1990), 185-192.

[7] V. Pescar, On some integral operations which preserve the univalence, Journal of Mathematics, Vol. XXX(1997), pp. 1-10, Punjab University. 